\title{
Acthar gel in the treatment of nephrotic syndrome: a multicenter retrospective case series
}

Arvind Madan ${ }^{1}$, Snezana Mijovic-Das², Ana Stankovic ${ }^{3}$, Geoffrey Teehan ${ }^{4}$, Amber S. Milward ${ }^{1}$ and Anupa Khastgir ${ }^{5^{*}}$

\begin{abstract}
Background: Current first-line anti-proteinuric treatments for nephrotic syndrome (NS) do not produce an effective response in all patients and are not tolerated by some patients. Additional effective and tolerable treatment options in NS are strongly needed. This retrospective case series is the largest to date to examine Acthar gel (adrenocorticotropic hormone, ACTH) in patients with varied-etiology NS.
\end{abstract}

Methods: This multicenter retrospective case series included adult patients with NS $(N=44)$ treated with Acthar gel at 6 clinical practices. NS etiologies included idiopathic focal segmental glomerulosclerosis (FSGS, 15), idiopathic membranous nephropathy (iMN, 11), IgA nephropathy (IgAN, 5), diabetic nephropathy (DN, 4), systemic lupus erythematosus class $\vee$ membranous lupus nephritis $(M L N, 2)$, minimal change disease $(M C D, 2)$, membranoproliferative glomerulonephritis (MPGN, 1), fibrillary glomerulonephritis (FGN, 1), and unbiopsied NS (3). Proteinuria response was assessed as percent reduction from baseline and percent of patients meeting complete remission (final proteinuria $<500 \mathrm{mg} / \mathrm{d}$ ), partial remission ( $\geq 50 \%$ reduction in proteinuria from baseline and final proteinuria $500-3500 \mathrm{mg} / \mathrm{d}$ ), clinical response ( $\geq 30 \%$ reduction in proteinuria from baseline that did not meet criteria for complete or partial remission), and no response (failed to meet remission or clinical response criteria) following Acthar gel therapy. Safety and tolerability were examined using adverse event (AE) frequency reported by patients or treating nephrologists and frequency of early discontinuation of treatment due to AEs.

Results: $68.2 \%$ (30/44) of patients had received prior NS treatment with immunosuppressive or cytotoxic therapies. Thirty-seven patients completed Acthar gel treatment. Seven patients (15.9\%) had early termination due to AEs, including weight gain (2), hypertension (2), edema (1), fatigue (1), seizures (1) and for reasons not stated (2). Proteinuria reduction $\geq 30 \%$ was shown in $81.1 \%(30 / 37)$ of patients and $62.2 \%(23 / 37)$ showed $\geq 50 \%$ proteinuria reduction. Proteinuria responses were greatest in MCD ( $n=2 / 2$ complete remission), MLN ( $n=2 / 2$ partial remission), MPGN ( $n=1 / 1$ partial remission), FSGS $(n=12 / 15$ [80.0\%] partial remission or clinical response), and iMN ( $n=8 / 11$ [72.7\%] complete remission, partial remission, or clinical response).

Conclusions: Acthar gel may meet an important treatment need in patients with treatment-resistant NS in response to first-line therapies, patients unable to tolerate first-line therapies, and in patients with advanced disease.

Keywords: ACTH, Acthar gel, Nephrotic syndrome, Proteinuria

\footnotetext{
* Correspondence: akhastgir@khastgirmedical.com

Arvind Madan and Anupa Khastgir are Co-first authors

${ }^{5}$ Nephrology Practice, 3366 NW Expressway, Bldg D, Suite 280, Oklahoma

City, OK 73112, USA

Full list of author information is available at the end of the article
} 


\section{Background}

Patients with nephrotic syndrome (NS) show a combination of clinical and laboratory features of renal diseases characterized by heavy proteinuria, hypoalbuminemia, and peripheral edema, with hyperlipidemia also frequently seen. Effective treatment in patients who experience treatment resistance or relapse following initial immunosuppressive treatment with steroids or cytotoxic drugs is an ongoing challenge across NS etiologies [1-8]. There remains a strong need for effective, tolerable treatments for patients with treatment-resistant NS, particularly without the renal and extra-renal toxicities associated with many first- and second-line therapies [9]. One such treatment is H.P. Acthar ${ }^{\circledR}$ Gel (adrenocorticotropic hormone, repository corticotropin injection, Mallinckrodt ARD Inc., Hazelwood, $\mathrm{MO})$, FDA-approved in the US to induce diuresis or remission of proteinuria in NS without uremia of the idiopathic type or that due to lupus erythematosus [10].

Adrenocorticotropic hormone (ACTH) treatment for NS emerged in the US in the 1950s and was shown to be effective for reduction or remission of proteinuria, depending on the dose and duration of ACTH treatment $[11,12]$. However, by the late 1960s ACTH had largely been replaced by steroids in NS treatment due to the convenience of oral dosing and the belief that they had similar mechanisms of action and treatment effects [13]. Acthar gel treatment for NS, the only FDA-approved ACTH treatment in the US, has recently re-emerged [14-20].

Though current clinical data are limited, proteinuria reduction resulting in complete or partial remission following Acthar gel treatment has been shown in patients with idiopathic membranous nephropathy (iMN), idiopathic focal segmental glomerulosclerosis (FSGS), IgA nephropathy (IgAN), minimal change disease (MCD), and diabetic nephropathy (DN) [14-20]. Treatment regimens of $80 \mathrm{U}$ Acthar gel twice weekly for 6 months were commonly used. One exception involved patients with iMN who received either a $40 \mathrm{U}$ or $80 \mathrm{U}$ dose twice weekly for either 3 months or 6 months, showing that greater reduction in proteinuria was associated with greater cumulative Acthar gel dose [19]. Additionally, patients with advanced DN were treated with Acthar gel $16 \mathrm{U}$ or $32 \mathrm{U}$ daily for 6 months [17].

Our retrospective case series is the largest published to date to examine the efficacy and safety of Acthar gel treatment in patients with varied-etiology NS who have typically failed multiple previous therapies. Proteinuria reduction as well as frequency of adverse events (AEs) in patients treated with Acthar gel within clinical practices were evaluated.

\section{Methods}

\section{Patients}

Patients eligible for inclusion were diagnosed with NS, $\geq 18$ years old, received Acthar gel treatment for $\geq 6$ months, and had assessment of either 24-h proteinuria level (mg/d) or urine protein:creatinine ratio (UPCR; g/g converted to $\mathrm{mg} / \mathrm{d}$ ) prior to and following 6 months of Acthar gel treatment. Patients did not have to meet a pre-specified level of proteinuria at baseline to be included in the study. NS etiologies within the patient cohort included idiopathic NS due to FSGS, MN, IgAN, MCD, membranoproliferative glomerulonephritis (MPGN), fibrillary glomerulonephritis (FGN), and 3 unbiopsied NS patients. Additionally, patients with systemic lupus erythematosus (SLE) class V membranous lupus nephritis (MLN), and patients receiving off-label treatment for NS due to DN were included. With the exception of the 3 unbiopsied patients, all patients had biopsy-confirmed NS etiology. The patient cohort represents all patients treated with Acthar gel from the participating clinical practices who met the specified inclusion criteria.

\section{Ethics}

This multicenter, retrospective case series of prescriptionbased treatment with Acthar gel for NS included 6 US clinical practices. The study received institutional review board exemption from the New England Institutional Review Board. All data reported in this paper have be de-identified in order to protect patient confidentiality.

\section{Data reviewed}

Clinical records were reviewed for demographic and clinical characteristics, including NS etiology, prior immunosuppressive or cytotoxic treatments, and levels of proteinuria, serum albumin, and total cholesterol. Hypoalbuminemia was defined as $<3.5 \mathrm{~g} / \mathrm{dL}$. Renal function was evaluated using serum creatinine (SCr) level and renal insufficiency was defined as $\mathrm{SCr}>1.3 \mathrm{mg} / \mathrm{dL}$. Acthar gel treatment dosing regimen and concomitant medications, including angiotensin II receptor blockers (ARBs), angiotensin-converting-enzyme inhibitors (ACEIs), and immunosuppressive and cytotoxic drugs, were documented.

Post-treatment proteinuria level and percentage reduction in proteinuria from baseline were examined. Consistent with prior studies, complete remission was defined as final proteinuria $<500 \mathrm{mg} / \mathrm{d}$, and partial remission was defined as $\geq 50 \%$ reduction in proteinuria from baseline and final proteinuria 500-3500 mg/d, with examination of preserved or improved renal function as indicated by $\mathrm{SCr}$ that does not worsen $>25 \%$ from baseline [14-16]. Clinical response was defined as $\geq 30 \%$ reduction in proteinuria from baseline that did not meet criteria for complete or partial remission. Inclusion of the clinical response outcome aligns with the treatment suggestion for calcineurin inhibitors (CNIs) in patients with iMN, stating therapy should be continued in patients showing an initial substantial proteinuria reduction of $30-50 \%$ at $4-6$ months treatment 
because the optimal treatment duration with calcineurin inhibitors is unknown and the patient's response suggests possible further proteinuria improvement with continued therapy [2]. The clinical response definition is also consistent with the clinical practice of the current study's treating nephrologists when determining the duration of a trial of treatment in patients. That is, treatment is extended following a $\geq 30 \%$ reduction in proteinuria because this degree of improvement has been experienced as a clinically meaningful change for patients. Patients showing no response failed to meet remission or clinical response criteria.

The frequency of AEs and the frequency of early discontinuation of treatment due to AEs were documented. Patients with an early termination of treatment without a stated reason were included in the count of early termination due to AEs.

\section{Data analysis}

Categorical variables were summarized using counts and percentages. Descriptive statistics summarized continuous variables. Paired $t$-tests examined change from baseline to post-Acthar gel therapy in proteinuria level, serum albumin, and total cholesterol. Between-group t-tests were used to compare percent reduction in proteinuria in patients who showed renal insufficiency at baseline versus patients who did not show renal insufficiency. Statistical analyses were performed using SAS (version 9.1; SAS Institute, Cary, NC).

\section{Results}

\section{Study participants}

Characteristics of patients by NS etiology group are presented in Table 1 . Cases included 44 patients across NS etiologies FSGS $(n=15)$, iMN $(n=11), \operatorname{IgAN}(n=5), \mathrm{DN}$ $(n=4), \operatorname{MLN}(n=2), \operatorname{MCD}(n=2), \operatorname{MPGN}(n=1), \mathrm{FGN}$, $(n=1)$, and 3 unbiopsied NS patients. The majority
(30/44; $68.2 \%$ ) had failed $\geq 1$ prior immunosuppressive or cytotoxic therapy, and 20 of 44 (45.5\%) had failed $\geq 2$ prior immunosuppressive and/or cytotoxic treatments. All patients received Acthar gel $80 \mathrm{U}$ twice weekly, with the exception of 1 patient with iMN who received $40 \mathrm{U}$ twice weekly. Continuing treatment with standard care ARB and/or ACEI maximum blockade was received by $36 / 44(81.8 \%)$ patients (Tables $2,3,4$ and 5; 12/15 FSGS, 9/11 iMN, 5/5 IgAN, 4/4 DN, 2/2 MLN, 2/2 MCD, 1/1 MPGN, 0/1 FGN, 1/3 unbiopsied NS) and dosing was maintained throughout the Acthar gel treatment period unless the patient required dose modification due to AEs.

\section{Total group treatment response}

There was significant proteinuria reduction from baseline to post-Acthar gel treatment $(n=40$; mean reduction $3984.8 \pm 4069.1 \mathrm{mg} / \mathrm{d}, P<0.0001)$. Total cholesterol showed significant decline from baseline to post-Acthar gel therapy $(n=21$; mean reduction $38.3 \pm 58.8 \mathrm{mg} / \mathrm{dL}$, $P=0.007)$. Mean serum albumin at baseline indicated hypoalbuminemia $(n=40,3.0 \pm 0.8 \mathrm{~g} / \mathrm{dL}$; range $1.4-4.0 \mathrm{~g} / \mathrm{dL})$, and significant improvement was shown post-Acthar gel therapy $(n=35$; mean improvement $0.53 \pm 0.6 \mathrm{~g} / \mathrm{dL}$, $P<0.0001)$.

Acthar gel treatment was completed by 37 patients, and 7 (15.9\%) patients had early termination of treatment due to AEs. Among the 37 treatment completers, $81.1 \%(30 / 37)$ showed $\geq 30 \%$ proteinuria reduction, and $62.2 \%(23 / 37)$ showed $\geq 50 \%$ proteinuria reduction. Proteinuria remission was shown by $56.8 \%(21 / 37)$ of patients, either complete $(n=4,10.8 \%)$ or partial $(n=$ 17, $45.9 \%$ remission. Inclusion of clinical response patients $(n=9)$ resulted in $81.1 \%(30 / 37)$ of patients showing substantial proteinuria reduction. Of these patients, $80 \%(24 / 30)$ had failed $\geq 1$ and $53.3 \%(16 / 30)$

Table 1 Demographic and clinical characteristics of NS patients $(N=44)$ treated with Acthar gel

\begin{tabular}{lllll}
\hline NS etiology & Age \pm SD, years & Gender, $n(\%)$ female & Race/ethnicity, $n(\%)$ White & Previous IST/CT, $n(\%)$ yes \\
\hline FSGS $(n=15)$ & $53.3 \pm 12.9$ & $7(47)$ & $12(80)$ & $12(80)$ \\
iMN $(n=11)$ & $53.6 \pm 18.9$ & $4(36)$ & $10(91)$ & $10(91)$ \\
IgAN $(n=5)$ & $35.0 \pm 8.4$ & $2(40)$ & $4(80)$ & $1(20)$ \\
DN $(n=4)$ & $54.0 \pm 19.9$ & $2(50)$ & $4(100)$ & 0 \\
MLN $(n=2)$ & $37.5 \pm 4.9$ & $1(50)$ & 0 & $2(100)$ \\
MCD $(n=2)$ & $33.5 \pm 13.4$ & $2(100)$ & $1(100)$ & $2(100)$ \\
FGN $(n=1)$ & 63.0 & 0 & $1(100)$ & $1(100)$ \\
MPGN $(n=1)$ & 22.0 & $1(100)$ & $2(67)$ & 0 \\
Other $^{a}(n=3)$ & $55.7 \pm 6.1$ & $2(67)$ & & $2(67)$
\end{tabular}

Abbreviations: CT cytotoxic therapy, DN diabetic nephropathy, FGN fibrillary glomerulonephritis, FSGS idiopathic focal segmental glomerulosclerosis, IgAN IgA nephropathy, iMN idiopathic membranous nephropathy, IST immunosuppressive therapy, MCD minimal change disease, MLN membranous lupus nephritis (class V), MPGN membranoproliferative glomerulonephritis, NS nephrotic syndrome

a"Other" includes 3 patients with unbiopsied NS 
Table 2 Proteinuria reduction and treatment response in patients with FSGS treated with Acthar gel

\begin{tabular}{|c|c|c|c|c|c|c|}
\hline \multirow[t]{4}{*}{ Patient } & \multirow[t]{4}{*}{ Previous IST/CT } & \multirow{4}{*}{$\begin{array}{l}\text { Concurrent } \\
\text { medications }\end{array}$} & \multirow{4}{*}{$\begin{array}{l}\text { Serum } \\
\text { albumin } \\
\text { Pre-Acthar } \\
\text { Post-Acthar } \\
(\mathrm{g} / \mathrm{dL})\end{array}$} & $\mathrm{SCr}$ & Proteinuria & \multirow{4}{*}{$\begin{array}{l}\text { Treatment } \\
\text { response }\end{array}$} \\
\hline & & & & Pre-Acthar & Pre-Acthar & \\
\hline & & & & \multirow{2}{*}{$\begin{array}{l}\text { Post-Acthar } \\
\% \text { change } \\
\text { (mg/dL) }\end{array}$} & \multirow{2}{*}{$\begin{array}{l}\text { Post-Acthar } \\
\% \text { change } \\
(\mathrm{mg} / \mathrm{d})\end{array}$} & \\
\hline & & & & & & \\
\hline \multirow[t]{3}{*}{1} & \multirow{3}{*}{$\begin{array}{l}\text { Prednisone, cyclosporine, } \\
\text { cyclophosphamide }\end{array}$} & \multirow[t]{3}{*}{ ACEl, cyclosporine } & 1.7 & 1.7 & 6700 & \multirow[t]{3}{*}{ Partial remission } \\
\hline & & & \multirow[t]{2}{*}{2.7} & 1.6 & 3300 & \\
\hline & & & & -5.9 & -50.7 & \\
\hline \multirow[t]{3}{*}{2} & \multirow{3}{*}{$\begin{array}{l}\text { Prednisone, cyclosporine, } \\
\text { MMF, tacrolimus, rituximab }\end{array}$} & \multirow[t]{3}{*}{ Tacrolimus, MMF } & 3.0 & 0.9 & 5800 & \multirow[t]{3}{*}{ Partial remission } \\
\hline & & & \multirow[t]{2}{*}{3.4} & 0.8 & 2016 & \\
\hline & & & & -11.1 & -65.2 & \\
\hline \multirow[t]{3}{*}{3} & \multirow[t]{3}{*}{ Prednisone } & \multirow[t]{3}{*}{ None } & 3.2 & 2.2 & 5000 & \multirow[t]{3}{*}{ Clinical response } \\
\hline & & & \multirow[t]{2}{*}{3.8} & 2.2 & 3422 & \\
\hline & & & & 0 & -31.6 & \\
\hline \multirow[t]{3}{*}{4} & \multirow[t]{3}{*}{ None } & ARB & 3.9 & 4.8 & 7900 & Partial remission \\
\hline & & & 4.0 & 6.7 & 2300 & \\
\hline & & & & 39.6 & -70.9 & \\
\hline 5 & None & ARB & 3.2 & 3.2 & 3840 & Early termination \\
\hline & & & NA & NA & NA & \\
\hline & & & & NA & NA & \\
\hline 6 & Prednisone, cyclosporine & ARB, ACEI cyclosporine & 2.8 & 3.0 & 7500 & Partial remission \\
\hline & & & 3.9 & 2.5 & 1768 & \\
\hline & & & & -16.7 & -76.4 & \\
\hline 7 & Prednisone, cyclosporine & ARB, ACEl cyclosporine & 3.1 & 1.1 & 5280 & Clinical response \\
\hline & & & 3.6 & 1.3 & 3560 & \\
\hline & & & & 18.2 & -32.6 & \\
\hline 8 & None & ACEI & NA & 1.2 & 4000 & Partial remission \\
\hline & & & NA & 1.1 & 765 & \\
\hline & & & & -8.3 & -80.9 & \\
\hline 9 & Prednisolone, methotrexate & ACEI, prednisolone & 3.1 & 1.6 & 9306 & Partial remission \\
\hline & & & 3.3 & 2.0 & 2773 & \\
\hline & & & & 25.0 & -70.2 & \\
\hline 10 & MMF & ACEI, MMF & NA & 4.4 & 2830 & Clinical response \\
\hline & & & 4.0 & 5.0 & 1629 & \\
\hline & & & & 13.6 & -42.4 & \\
\hline 11 & Prednisone & ARB & 3.5 & 2.4 & 3500 & Partial remission \\
\hline & & & 3.7 & 3.1 & 750 & \\
\hline & & & & 29.2 & -78.6 & \\
\hline 12 & Prednisone & ACEI & 3.8 & 1.5 & 5700 & Early termination \\
\hline & & & 4.0 & 1.2 & $1500^{a}$ & \\
\hline & & & & -20.0 & -73.7 & \\
\hline 13 & Prednisone, cyclosporine & None & 3.2 & 3.1 & 3250 & Clinical response \\
\hline & & & 3.5 & 4.1 & 2073 & \\
\hline & & & & 32.3 & -36.2 & \\
\hline
\end{tabular}


Table 2 Proteinuria reduction and treatment response in patients with FSGS treated with Acthar gel (Continued)

\begin{tabular}{lllllll}
\hline 14 & Prednisone, cyclosporine & ACEl & 3.7 & 2.5 & 2500 & 1246 \\
\\
\end{tabular}

Abbreviations: $A C E l$ angiotensin-converting-enzyme inhibitor, $A R B$ angiotensin II receptor blockers, CT cytotoxic therapy, FSGS idiopathic focal segmental glomerulosclerosis, IST immunosuppressive therapy, MMF mycophenolate mofetil, NA not available

a Post-Acthar gel assessment occurred following 4 months of treatment

had failed $\geq 2$ prior immunosuppressive or cytotoxic therapies.

Among the 44 patients, 26 (59.1\%) showed SCr $>1.3 \mathrm{mg} /$ $\mathrm{dL}$ at baseline. There was a greater mean percent proteinuria reduction in patients with $\mathrm{SCr} \leq 1.3 \mathrm{mg} / \mathrm{dL}$ ( $n=14 ; 72.2 \pm 26.9 \%$ reduction) compared with patients showing $\mathrm{SCr}>1.3 \mathrm{mg} / \mathrm{dL}(n=22 ; 41.0 \pm 29.7 \%$ reduction, $P=0.0031)$. Similarly, among patients showing complete or partial remission or clinical response, greater mean percent proteinuria reduction occurred in patients without renal function impairment $(n=13 ; 77.5 \pm 19.0 \%$ reduction) compared with $\mathrm{SCr}>1.3 \mathrm{mg} / \mathrm{dL}(n=16 ; 55.6 \pm$ $16.9 \%$ reduction, $P=0.0029)$.

\section{Treatment response by NS etiology}

The percentage of patients showing complete remission, partial remission, or clinical response to Acthar gel treatment varied across NS etiologies (Fig. 1). The highest proteinuria responses were seen in patients with MCD ( $n=2 / 2$ complete remission), MLN ( $n=2 / 2$ partial remission), MPGN ( $n=1 / 1$ partial remission), FSGS ( $n=12 / 15$ [80.0\%] partial remission or clinical response), and iMN ( $n=8 / 11$ [72.7 \%] complete or partial remission or clinical response). Lower proteinuria responses were seen in patients with IgAN $(n=2 / 5$ [40.0\%] partial remission or clinical response) and DN $(n=1 / 4$ [25 \%] clinical response). The single patient with FGN showed no response. Within the "Other" category of unbiopsied NS patients, 1 patient showed partial remission and 1 patient showed clinical response.

\section{FSGS}

Following Acthar gel, $86.7 \%$ (13/15) of patients showed $\geq 30 \%$ proteinuria reduction and $60 \%$ (9/15) showed $\geq 50 \%$ proteinuria reduction (Table 2 ). There was significant proteinuria reduction from baseline to postActhar gel treatment $(n=14$; mean reduction $3021.7 \pm$ $1970.6 \mathrm{mg} / \mathrm{d}, P<0.0001)$. No patient showed complete remission; however, 9 (60\%) showed partial remission, and $4(26.7 \%)$ showed clinical responses ranging from $31.6-42.4 \%$ proteinuria reduction. One patient showed no response, and 2 patients had early termination. The early termination for 1 patient was due to increased swelling; no reason was provided for the second patient, who achieved partial remission prior to termination of treatment. Renal insufficiency at baseline due to $\mathrm{SCr}>1.3 \mathrm{mg} / \mathrm{dL}$ was shown in $12 / 15$ (80 \%) patients. Among the Acthar gel treatment responders, worsening $\mathrm{SCr}>25 \%$ was shown in 2 patients with partial remission and 1 patient with clinical response and all 3 patients had $\mathrm{SCr}>1.3$ at baseline (Table 2). Total cholesterol significantly declined from baseline $(n=8$; mean reduction $32.5 \pm 28.9 \mathrm{mg} / \mathrm{dL}, P<0.02)$, and serum albumin significantly increased $(n=11$; mean increase $0.39 \pm$ $0.4 \mathrm{~g} / \mathrm{dL}, P=0.009)$. Hypoalbuminemia was shown by $8 / 15$ patients $(53.3 \%$ ) at baseline (range $1.7-3.2 \mathrm{~g} / \mathrm{dL}$ ), and improved in 7 of these patients by post-treatment (range $2.7-3.9 \mathrm{~g} / \mathrm{dL}$ ).

\section{iMN}

Proteinuria reduction $\geq 50 \%$ occurred in $72.7 \%(8 / 11)$ of patients with iMN (Table 3). There was significant proteinuria reduction from baseline to post-Acthar gel treatment $(n=10$; mean reduction $4245.5 \pm 4085.5 \mathrm{mg} / \mathrm{d}$, $P=0.009)$. Two patients (18.2\%) showed complete remission, 4 (36.4 \%) showed partial remission, and 2 (18.2\%) showed clinical responses ranging from 51.5-60.0 \% proteinuria reduction. Two patients showed no response (1 patient was diagnosed with iMN and FSGS), and 1 patient had early termination due to an AE involving fatigue. $\mathrm{SCr}$ $>1.3 \mathrm{mg} / \mathrm{dL}$ was found in $5 / 11$ patients $(45.5 \%)$ at baseline. The mean reduction in total cholesterol $(n=5 ; 9.4 \pm$ $10.2 \mathrm{mg} / \mathrm{dL})$ and increase in serum albumin $(n=9 ; 0.52 \pm$ $0.7 \mathrm{~g} / \mathrm{dL})$ were not significant $(P>0.06)$. Hypoalbuminemia was shown by $6 / 11$ patients $(54.5 \%)$ at baseline (range 1.4-3.3 g/dL), and 5 of these patients showed improvement post-treatment (range $2.1-3.4 \mathrm{~g} / \mathrm{dL}$ ).

\section{IgAN}

Proteinuria reduction $\geq 30 \%$ occurred in $60 \%(3 / 5)$ of patients and $40 \%(2 / 5)$ showed $\geq 50 \%$ proteinuria reduction (Table 4$)$. The mean proteinuria reduction $(n=4)$ 
Table 3 Proteinuria reduction and treatment response in patients with iMN treated with Acthar gel

\begin{tabular}{|c|c|c|c|c|c|c|}
\hline \multirow[t]{4}{*}{ Patient } & \multirow[t]{4}{*}{ Previous IST/CT } & \multirow{4}{*}{$\begin{array}{l}\text { Concurrent } \\
\text { medications }\end{array}$} & \multirow{4}{*}{$\begin{array}{l}\text { Serum } \\
\text { albumin } \\
\text { Pre-Acthar } \\
\text { Post-Acthar } \\
(\mathrm{g} / \mathrm{dL})\end{array}$} & $\mathrm{SCr}$ & Proteinuria & \multirow[t]{4}{*}{ Treatment response } \\
\hline & & & & Pre-Acthar & Pre-Acthar & \\
\hline & & & & \multirow{2}{*}{$\begin{array}{l}\text { Post-Acthar } \\
\% \text { change } \\
\text { (mg/dL) }\end{array}$} & \multirow{2}{*}{$\begin{array}{l}\text { Post-Acthar } \\
\% \text { change } \\
\text { (mg/d) }\end{array}$} & \\
\hline & & & & & & \\
\hline \multirow[t]{3}{*}{1} & \multirow[t]{3}{*}{ Prednisone, cyclophosphamide, rituximab } & \multirow[t]{3}{*}{ ARB, ACEI } & 2.7 & 1.3 & 13,600 & \multirow[t]{3}{*}{ Clinical response } \\
\hline & & & \multirow[t]{2}{*}{3.2} & 1.3 & 6600 & \\
\hline & & & & 0 & -51.5 & \\
\hline \multirow[t]{3}{*}{2} & \multirow[t]{3}{*}{ Prednisone, IVMP } & \multirow[t]{3}{*}{ Prednisone } & 1.4 & 1.2 & 6354 & \multirow[t]{3}{*}{ Partial remission } \\
\hline & & & \multirow[t]{2}{*}{3.3} & 1.1 & 1000 & \\
\hline & & & & -8.3 & -84.3 & \\
\hline \multirow[t]{3}{*}{3} & \multirow[t]{3}{*}{ None } & \multirow[t]{3}{*}{ None } & 1.5 & 2.5 & 15,400 & \multirow[t]{3}{*}{ Partial remission } \\
\hline & & & \multirow[t]{2}{*}{3.1} & 1.9 & 2376 & \\
\hline & & & & -24.0 & -84.6 & \\
\hline \multirow[t]{3}{*}{4} & \multirow[t]{3}{*}{ Prednisone, cyclosporine } & \multirow[t]{3}{*}{ ACE } & 1.6 & 1.9 & 10,000 & \multirow[t]{3}{*}{ Clinical response } \\
\hline & & & \multirow[t]{2}{*}{2.1} & 1.9 & 4000 & \\
\hline & & & & 0 & -60.0 & \\
\hline 5 & Prednisone & ARB, ACEI & 3.8 & 1.9 & 4000 & No response \\
\hline & & & 3.7 & 2.0 & 3475 & \\
\hline & & & & 5.3 & -13.1 & \\
\hline 6 & Tacrolimus & ACEI & 3.3 & 1.0 & 5500 & Complete remission \\
\hline & & & 3.4 & 0.9 & 349 & \\
\hline & & & & -10.0 & -93.7 & \\
\hline 7 & Prednisone, tacrolimus, chlorambucil & ARB & 3.9 & 0.9 & 2400 & Complete remission \\
\hline & & Tacrolimus & 4.0 & 1.0 & 163 & \\
\hline & & & & -11.1 & -93.2 & \\
\hline 8 & Prednisone, cyclosporine & ACEI & 3.2 & 1.3 & 3070 & Early termination \\
\hline & & & NA & NA & NA & \\
\hline & & & & NA & NA & \\
\hline 9 & Prednisone, cyclosporine & ACEI & 3.8 & 2.1 & 1930 & Partial remission \\
\hline & & & 3.7 & 2.2 & 728 & \\
\hline & & & & 4.8 & -62.3 & \\
\hline 10 & Prednisone, tacrolimus, cyclophosphamide & ARB & 3.5 & 1.3 & 5210 & Partial remission \\
\hline & & & 3.7 & 1.6 & 1780 & \\
\hline & & & & 23.1 & -65.8 & \\
\hline 11 & Prednisone, IVMP, cyclophosphamide & ACEI & NA & 3.3 & 5132 & No response \\
\hline & & & NA & 3.8 & 6600 & \\
\hline & & & & 15.2 & 28.6 & \\
\hline
\end{tabular}

Abbreviations: $A C E$ langiotensin-converting-enzyme inhibitor, $A R B$ angiotensin II receptor blockers, $C T$ cytotoxic therapy, iMN idiopathic membranous nephropathy, IST immunosuppressive therapy, IVMP intravenous methylprednisolone, NA not available

was $2917.0 \pm 4225.4 \mathrm{mg} / \mathrm{d}$. Two patients showed partial remission and 1 patient showed clinical response (36.4\% proteinuria reduction). One patient showed no response and 2 patients had early termination. Early termination for 1 patient was due to AEs of weight gain and hypertension, and for 1 patient was stated as a patient decision. This latter patient showed partial remission, with
$92 \%$ proteinuria reduction before treatment termination. $\mathrm{SCr}>1.3 \mathrm{mg} / \mathrm{dL}$ occurred in $2 / 5$ patients (40\%) at baseline. Total cholesterol and serum albumin were available for 2 patients. Total cholesterol decreased from $250 \mathrm{mg} / \mathrm{dL}$ to $230 \mathrm{mg} / \mathrm{dL}$ and serum albumin increased from $3.0 \mathrm{~g} / \mathrm{dL}$ to $4.0 \mathrm{~g} / \mathrm{dL}$ in 1 patient whereas the second patient had increased total cholesterol from 
Table 4 Proteinuria reduction and treatment response in patients with IgA nephropathy/diabetic nephropathy treated with Acthar

\begin{tabular}{|c|c|c|c|c|c|c|}
\hline \multirow[t]{3}{*}{ Patient } & \multirow[t]{3}{*}{ Previous IST/CT } & \multirow{3}{*}{$\begin{array}{l}\text { Concurrent } \\
\text { medications }\end{array}$} & Serum albumin & $\mathrm{SCr}$ & Proteinuria & \multirow{3}{*}{$\begin{array}{l}\text { Treatment } \\
\text { response }\end{array}$} \\
\hline & & & Pre-Acthar & Pre-Acthar & Pre-Acthar & \\
\hline & & & $\begin{array}{l}\text { Post-Acthar } \\
\text { (g/dL) }\end{array}$ & $\begin{array}{l}\text { Post-Acthar } \\
\% \text { change } \\
(\mathrm{mg} / \mathrm{dL})\end{array}$ & $\begin{array}{l}\text { Post-Acthar } \\
\% \text { change } \\
\text { (mg/d) }\end{array}$ & \\
\hline \multicolumn{7}{|l|}{$\lg A N$} \\
\hline \multirow[t]{3}{*}{1} & \multirow[t]{3}{*}{ None } & \multirow[t]{3}{*}{ ARB, ACEI } & 3.9 & 2.8 & 4000 & \multirow[t]{3}{*}{ Early termination } \\
\hline & & & \multirow[t]{2}{*}{ NA } & NA & NA & \\
\hline & & & & NA & NA & \\
\hline \multirow[t]{3}{*}{2} & \multirow[t]{3}{*}{ None } & \multirow[t]{3}{*}{ ARB, ACEI } & 3.9 & 1.4 & 2674 & \multirow[t]{3}{*}{ Clinical response } \\
\hline & & & \multirow[t]{2}{*}{ NA } & 1.5 & 1700 & \\
\hline & & & & 7.1 & -36.4 & \\
\hline \multirow[t]{3}{*}{3} & \multirow[t]{3}{*}{ None } & \multirow[t]{3}{*}{ ARB } & 4.0 & 1.3 & 2439 & \multirow[t]{3}{*}{ No response } \\
\hline & & & 3.6 & 1.3 & 2360 & \\
\hline & & & & 0 & -3.2 & \\
\hline \multirow[t]{3}{*}{4} & \multirow[t]{3}{*}{ None } & \multirow[t]{3}{*}{ ACEI } & 3.0 & 1.0 & 10000 & \multirow{3}{*}{$\begin{array}{l}\text { Early termination } \\
\text { (Partial remission) }\end{array}$} \\
\hline & & & 4.0 & 1.0 & $800^{\mathrm{a}}$ & \\
\hline & & & & 0 & -92.0 & \\
\hline \multirow[t]{3}{*}{5} & \multirow{3}{*}{$\begin{array}{l}\text { Prednisone, cyclophosphamide, } \\
\text { azathioprine }\end{array}$} & \multirow[t]{3}{*}{ ARB } & NA & 1.3 & 2230 & \multirow[t]{3}{*}{ Partial remission } \\
\hline & & & 4.2 & 1.3 & 815 & \\
\hline & & & & 0 & -63.5 & \\
\hline \multicolumn{7}{|l|}{ DN } \\
\hline \multirow[t]{3}{*}{1} & \multirow[t]{3}{*}{ None } & ACEI & 2.0 & 1.9 & 25000 & No response \\
\hline & & & 2.0 & 4.9 & 23000 & \\
\hline & & & & 157.9 & -8.0 & \\
\hline 2 & None & ARB, ACEI & 3.3 & 3.4 & 14000 & Early termination \\
\hline & & & 3.3 & 4.4 & $11600^{\mathrm{a}}$ & \\
\hline & & & & 29.4 & -17.1 & \\
\hline 3 & None & ACEI & 3.1 & 4.8 & 17570 & No response \\
\hline & & & 2.8 & 5.7 & 18886 & \\
\hline & & & & 18.8 & 7.5 & \\
\hline 4 & None & ACEI & 3.4 & 2.5 & 11000 & Clinical response \\
\hline & & & 3.5 & 2.5 & 6895 & \\
\hline & & & & 0 & -37.3 & \\
\hline
\end{tabular}

Abbreviations: $A C E$ langiotensin-converting-enzyme inhibitor, $A R B$ angiotensin II receptor blockers, $C T$ cytotoxic therapy, DN diabetic nephropathy, IgAN IgA nephropathy, IST immunosuppressive therapy, MMF mycophenolate mofetil, NA not available

a Post-Acthar gel assessment occurred following 3 months of treatment

$161 \mathrm{mg} / \mathrm{dL}$ to $242 \mathrm{mg} / \mathrm{dL}$ and decreased serum albumin from $4.0 \mathrm{~g} / \mathrm{dL}$ to $3.6 \mathrm{~g} / \mathrm{dL}$. One patient showed hypoalbuminemia at baseline $(3.0 \mathrm{~g} / \mathrm{dL})$ and improved posttreatment $(4.0 \mathrm{~g} / \mathrm{dL})$.

\section{DN}

Among 4 patients, $1(25 \%)$ showed $\geq 30 \%$ proteinuria reduction (37.3\%, Table 4). The mean proteinuria reduction $(n=4)$ was $1797.3 \pm 2267.3 \mathrm{mg} / \mathrm{d}$. Two patients had no response to treatment, including 1 patient with $\mathrm{DN}$ and FSGS who progressed to renal replacement therapy. One patient had early termination due to AEs involving weight gain and hypertension. All 4 patients showed $\mathrm{SCr}$ $>1.3 \mathrm{mg} / \mathrm{dL}$. There was a mean reduction in total cholesterol $(n=2 ; 104.5 \pm 13.4 \mathrm{mg} / \mathrm{dL})$ and in serum albumin ( $n=4 ; 0.05 \pm 0.2 \mathrm{~g} / \mathrm{dL})$. Hypoalbuminemia was shown by all 4 patients at baseline (range $2.0-3.4 \mathrm{~g} / \mathrm{dL}$ ) and improved to $3.5 \mathrm{~g} / \mathrm{dL}$ in 1 patient. 
Table 5 Proteinuria reduction and treatment response in patients treated with Acthar gel, by etiologic diagnosis

\begin{tabular}{|c|c|c|c|c|c|c|}
\hline \multirow[t]{3}{*}{ Patient } & \multirow[t]{3}{*}{ Previous IST/CT } & \multirow{3}{*}{$\begin{array}{l}\text { Concurrent } \\
\text { medications }\end{array}$} & Serum albumin & $\mathrm{SCr}$ & Proteinuria & \multirow[t]{3}{*}{ Treatment response } \\
\hline & & & Pre-Acthar & Pre-Acthar & Pre-Acthar & \\
\hline & & & $\begin{array}{l}\text { Post-Acthar } \\
\text { (g/dL) }\end{array}$ & $\begin{array}{l}\text { Post-Acthar } \\
\% \text { change } \\
\text { (mg/dL) }\end{array}$ & $\begin{array}{l}\text { Post-Acthar } \\
\% \text { change } \\
\text { (mg/d) }\end{array}$ & \\
\hline \multicolumn{7}{|l|}{ MLN } \\
\hline \multirow[t]{3}{*}{1} & \multirow[t]{3}{*}{ Prednisone, cyclophosphamide } & \multirow[t]{3}{*}{ ACEl, prednisone } & 1.8 & 1.0 & 8000 & \multirow[t]{3}{*}{ Partial remission } \\
\hline & & & 3.3 & 0.8 & 1089 & \\
\hline & & & & -20.0 & -86.4 & \\
\hline \multirow[t]{3}{*}{2} & \multirow[t]{3}{*}{ MMF } & \multirow[t]{3}{*}{ ACEI } & 1.7 & 1.0 & 19890 & \multirow[t]{3}{*}{ Partial remission } \\
\hline & & & 2.4 & 1.1 & 2454 & \\
\hline & & & & 10.0 & -87.7 & \\
\hline \multicolumn{7}{|l|}{ MCD } \\
\hline \multirow[t]{3}{*}{1} & \multirow[t]{3}{*}{ Prednisone } & \multirow[t]{3}{*}{ ACEI } & 3.7 & 0.9 & 2000 & \multirow[t]{3}{*}{ Complete remission } \\
\hline & & & 4.7 & 1.2 & 241 & \\
\hline & & & & 33.0 & -88.0 & \\
\hline \multirow[t]{3}{*}{2} & \multirow[t]{3}{*}{ Prednisone, cyclosporine } & \multirow[t]{3}{*}{ ACEI } & 2.1 & 1.0 & 15000 & \multirow[t]{3}{*}{ Complete remission } \\
\hline & & & 2.3 & 0.7 & 89 & \\
\hline & & & & -30.0 & -99.4 & \\
\hline \multirow[t]{3}{*}{ FGN } & \multirow[t]{3}{*}{ Prednisone, MMF, rituximab } & \multirow[t]{3}{*}{ MMF } & 1.4 & 5.6 & 13000 & \multirow[t]{3}{*}{ No response } \\
\hline & & & 3.4 & 9.0 & 10000 & \\
\hline & & & & 60.7 & -23.1 & \\
\hline \multirow[t]{3}{*}{ MPGN } & \multirow[t]{3}{*}{ None } & \multirow[t]{3}{*}{ ACEI } & 1.5 & 0.7 & 10000 & Partial remission \\
\hline & & & 3.3 & 0.8 & 2141 & \\
\hline & & & & 14.3 & -78.6 & \\
\hline OTHER ${ }^{a}$ & & & & & & \\
\hline 1 UNS & Prednisone & None & 3.5 & 1.6 & 3000 & Clinical response \\
\hline & & & 4.6 & 2.2 & 1600 & \\
\hline & & & & 37.5 & -46.7 & \\
\hline 2 UNS & Prednisone & ACEI & 3.5 & 1.7 & 4500 & Partial remission \\
\hline & & & 4.0 & 1.9 & 2000 & \\
\hline & & & & 11.8 & -55.6 & \\
\hline 3 UNS & None & None & 3.1 & 1.3 & 5500 & Early termination \\
\hline & & & 3.3 & 1.3 & NA & \\
\hline & & & & 0 & NA & \\
\hline
\end{tabular}

Abbreviations: $A C E$ l angiotensin-converting-enzyme inhibitor, $A R B$ angiotensin II receptor blockers, FGN fibrillary glomerulonephritis, $M C D$ minimal change disease, MLN membranous lupus nephritis (class V), MPGN membranoproliferative glomerulonephritis, NA not available, UNS unbiopsied nephrotic syndrome

a"Other" includes 3 patients with unbiopsied NS

\section{MLN}

Both patients with MLN showed $\geq 50 \%$ proteinuria reduction (86.4 \% and $87.7 \%$ ), and showed partial remission (Table 5). The mean proteinuria reduction $(n=2)$ was $12173.5 \pm 7442.3 \mathrm{mg} / \mathrm{d}$. The patients did not experience renal insufficiency, but they did show hypoalbuminemia at baseline (1.7 and $1.8 \mathrm{~g} / \mathrm{dL})$, which improved post-treatment (3.3 and $2.4 \mathrm{~g} / \mathrm{dL})$. There was a mean increase in serum albumin $(n=2 ; 1.10 \pm 0.6 \mathrm{~g} / \mathrm{dL})$.
Change in total cholesterol was not available for either patient.

\section{$M C D$}

Both patients with MCD showed $\geq 50 \%$ proteinuria reduction (88\% and 99.4\%), and showed complete remission (Table 5). The mean proteinuria reduction $(n=2)$ was $8335.0 \pm 9299.9 \mathrm{mg} / \mathrm{d}$. Renal insufficiency was not present but 1 patient showed a worsening of $\mathrm{SCr}$ from 


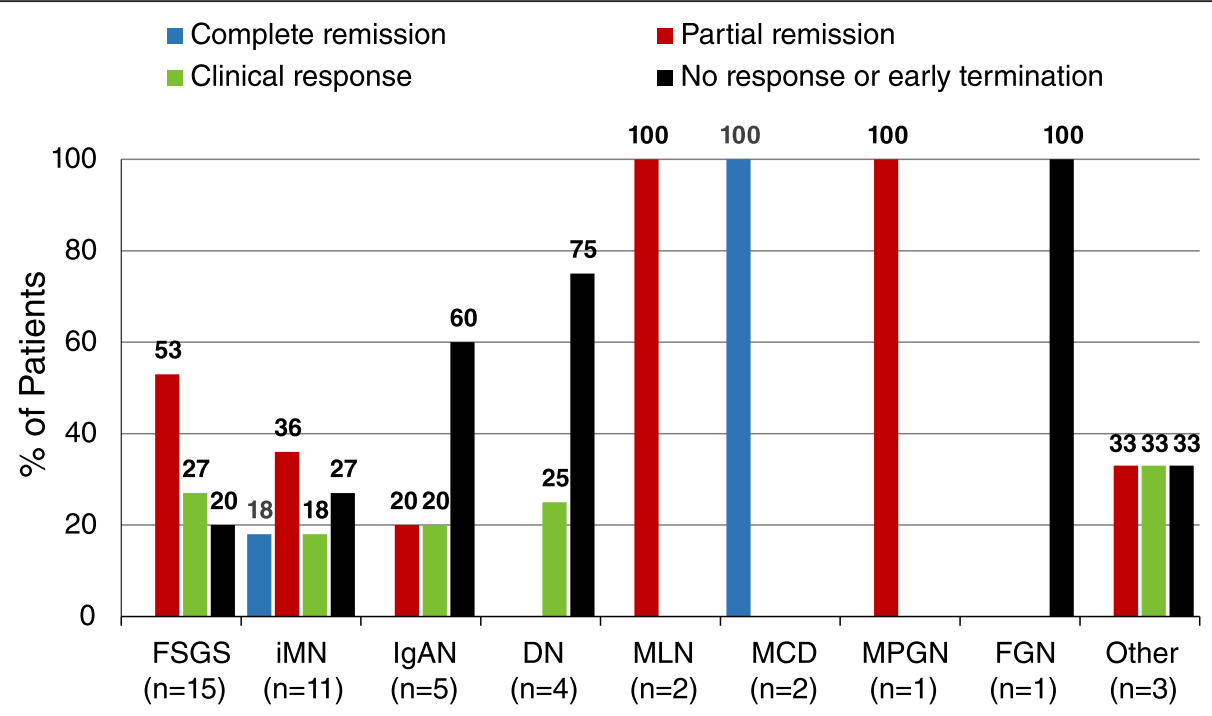

Fig. 1 Treatment response in patients with NS treated with Acthar gel, by etiologic diagnosis. "Other" includes 3 patients with unbiopsied NS. Abbreviations: DN, diabetic nephropathy; FGN, fibrillary glomerulonephritis; FSGS, idiopathic focal segmental glomerulosclerosis; IgAN, IgA nephropathy; iMN, idiopathic membranous nephropathy; MCD, minimal change disease; MLN, membranous lupus nephritis (class V); MPGN, membranoproliferative glomerulonephritis

0.9 at baseline to $1.2 \mathrm{mg} / \mathrm{dL}$ (33 \%). One patient showed hypoalbuminemia at baseline $(2.1 \mathrm{~g} / \mathrm{dL})$ with minimal improvement post-treatment $(2.3 \mathrm{~g} / \mathrm{dL})$. There was mean total cholesterol reduction $(n=2 ; 81.5 \pm 2.1 \mathrm{mg} / \mathrm{dL})$ and mean serum albumin increase $(n=2 ; 0.60 \pm 0.6 \mathrm{~g} / \mathrm{dL})$.

\section{FGN}

The patient with FGN showed no response to treatment (Table 5). The patient had advanced disease that required renal replacement therapy, and dialysis was initiated before starting Acthar gel therapy.

\section{MPGN}

The patient with MPGN showed partial remission, with $78.6 \%$ proteinuria reduction (Table 5). This patient did not show renal insufficiency but did show hypoalbuminemia at baseline $(1.5 \mathrm{~g} / \mathrm{dL})$ that improved post-treatment $(3.3 \mathrm{~g} / \mathrm{dL})$. Total cholesterol was reduced from $360 \mathrm{mg} / \mathrm{dL}$ to $180.0 \mathrm{mg} / \mathrm{dL}$.

\section{Unbiopsied NS}

Among the 3 patients with unbiopsied NS, 1 patient showed partial remission (55.6\% proteinuria reduction), and 1 patient showed clinical response $(46.7 \%$ proteinuria reduction) (Table 5). The third patient had early termination of treatment due to an $\mathrm{AE}$ involving seizures. The patients with partial remission and clinical response showed renal insufficiency at baseline and the patient with a clinical response showed worsening of $\mathrm{SCr}>25 \%$. Hypoalbuminemia was present in the patient with early termination. The patient with partial remission had a total cholesterol reduction of $100 \mathrm{mg} / \mathrm{dL}$.

\section{Safety and tolerability}

AEs during Acthar gel treatment were reported by $29.5 \%$ (13/44) of patients and included increased swelling, weight gain, hypertension, hyperglycemia, fatigue, dizziness, hypokalemia, upper respiratory infection, seizures, and decreased bone mineralization (Table 6). Early termination due to treatment-related AEs occurred in $15.9 \%$ (7/44) of patients. Among these 7 patients, 1 patient with IgAN terminated treatment early by "patient decision" and 1 patient with FSGS terminated treatment early without a specific reason provided. Both patients showed partial remission before early termination (Table 2 , Table 4 ).

\section{Discussion}

This retrospective case series is the largest published to date to examine the efficacy and safety of Acthar gel in the treatment of patients with NS of varying etiologies, $68.2 \%$ of whom had received prior NS treatment with immunosuppressive or cytotoxic therapies, who were receiving clinic-based prescription treatment. A significant proteinuria reduction was shown, and approximately $80 \%$ of patients who completed Acthar gel treatment showed a substantial proteinuria reduction of $\geq 30 \%$, including patients who met criteria for complete remission, partial remission, or clinical response. Most patients tolerated Acthar gel therapy well. The AEs were consistent with prior studies of Acthar gel in patients with NS, in which AEs were typically steroidlike, with most being mild to moderate in severity and transient [14-17].

The relative rarity of NS etiologies has contributed to the scarcity of large-scale, prospective, randomized, 
Table 6 Adverse events and early termination of Acthar gel treatment in patients with NS

\begin{tabular}{|c|c|c|c|}
\hline $\begin{array}{l}\text { Nephrotic syndrome } \\
\text { etiology }\end{array}$ & $\begin{array}{l}\text { Patients reporting } \\
\text { treatment-related AEs, } \\
n(\%)\end{array}$ & Treatment-related AEs & Early termination due to $\mathrm{AEs}^{\mathrm{a}}, n(\%)$ \\
\hline FSGS $(n=15)$ & $3(20 \%)$ & $\begin{array}{l}\text { Increased swelling }(n=1) \\
\text { Hyperglycemia }(n=2) \\
\text { Hypertension }(n=1) \\
\text { Weight gain }(n=1) \\
\text { Upper respiratory infections }(n=1)\end{array}$ & $\begin{array}{l}1(6.7 \%) \text { Edema } \\
1(6.7 \%) \text { Reason not given }\end{array}$ \\
\hline $\mathrm{iMN}(n=11)$ & $4(36.4 \%)$ & $\begin{array}{l}\text { Fatigue }(n=1) \\
\text { Dizziness }(n=1) \\
\text { Weight gain }(n=2) \\
\text { Hypokalemia }(n=1)\end{array}$ & 1 (9\%) Fatigue \\
\hline $\lg \mathrm{AN}(n=5)$ & $1(20 \%)$ & $\begin{array}{l}\text { Weight gain }(n=1) \\
\text { Hypertension }(n=1)\end{array}$ & $\begin{array}{l}1(20 \%) \text { Weight gain, hypertension } \\
1(20 \%) \text { Patient decision }\end{array}$ \\
\hline $\mathrm{DN}(n=4)$ & $3(75 \%)$ & $\begin{array}{l}\text { Weight gain }(n=2) \\
\text { Hypertension }(n=1) \\
\text { Hyperglycemia }(n=1) \\
\text { Decreased bone mineralization }(n=1)\end{array}$ & 1 (25 \%) Weight gain, hypertension \\
\hline $\operatorname{MLN}(n=2)$ & 0 & & 0 \\
\hline $\operatorname{MCD}(n=2)$ & 0 & & 0 \\
\hline FGN $(n=1)$ & 0 & & 0 \\
\hline $\operatorname{MPGN}(n=1)$ & 0 & & 0 \\
\hline Other $^{\mathrm{b}}(n=3)$ & $2(66.7 \%)$ & $\begin{array}{l}\text { Seizures }(n=1) \\
\text { Hyperglycemia }(n=1) \\
\text { Weight gain }(n=1) \\
\text { Hypertension }(n=1)\end{array}$ & 1 (33.3 \%) Seizures \\
\hline
\end{tabular}

Abbreviations: $A E s$ adverse events, DN diabetic nephropathy, FGN fibrillary glomerulonephritis, FSGS idiopathic focal segmental glomerulosclerosis, IgAN IgA nephropathy, $i M N$ idiopathic membranous nephropathy, MCD minimal change disease, MLN membranous lupus nephritis (SLE class V), MPGN membranoproliferative glomerulonephritis, UNS unbiopsied nephrotic syndrome

${ }^{a}$ Patients without a specific reason given for early termination of treatment were included in the count of early termination due to AEs

b"Other" includes 3 patients with unbiopsied NS

controlled trials on which to base treatment recommendations [1]. As a result, the majority $(67 \%)$ of the recommendations provided by the comprehensive Kidney Disease: Improving Global Outcomes (KDIGO) 2012 Clinical Practice Guideline for Glomerulonephritis were graded as a suggestion rather than recommendation and with an evidence quality rating of $\mathrm{C}$ or $\mathrm{D}$, indicating low to very low quality of evidence [1]. Evaluation of additional treatment options is urgently needed for patients with FSGS, iMN, IgAN, MCD, and MLN who are not responsive to the first-line treatments-typically corticosteroids, cyclophosphamide, CNIs, and mycophenolate mofetil (MMF)-or who are unable to tolerate the firstline treatments. For example, approximately $25 \%$ of patients with MCD have been shown to be steroid-resistant and approximately $30 \%$ of initial steroid responders show frequent relapses, and approximately one-third of MLN patients have been shown not to respond to the current American College of Rheumatology (ACR)-recommended initial treatment with prednisone plus $\operatorname{MMF}[4,7,8]$. While acknowledging retrospective case series study design limitations, the current large case series provides much-needed Acthar gel treatment response information in diverse patients, including patients with advanced disease and treatment-resistant NS. Half (53.3 \%) of the patients who showed proteinuria response to Acthar gel had failed $\geq 2$ prior immunosuppressive or cytotoxic therapies, and approximately half had impaired renal function. Among all patients and among patients who showed proteinuria reduction, patients with preserved renal function showed greater percent proteinuria reduction following Acthar gel treatment, indicating earlier treatment with Acthar gel may be especially beneficial.

Importantly, studies of patients with FSGS, iMN, and IgAN have indicated that partial remission and improved disease control are associated with better renal outcomes, even if patients relapse again $[6,21,22]$. Although complete remission is the ideal outcome, these studies suggest reduced proteinuria that does not meet complete remission criteria provides a meaningful treatment benefit compared with no improved disease control $[6,21,22]$. The optimal treatment duration for Acthar gel in patients with NS of varied etiology is not yet known. Our inclusion of the clinical response outcome identifying patients with substantial proteinuria reduction $\geq 30 \%$ is consistent with the suggestion that longer-duration treatment beyond 6 months is indicated in iMN patients receiving CNIs who show proteinuria reduction of $30-50 \%$, with the goal of achieving partial or complete remission with longerduration therapy [2]. Additionally, within the clinic, our 
experience with patients who show proteinuria reduction $\geq 30 \%$ is clinically meaningful improvement in the patient's report of feeling better. Longer-term treatment follow-up of these patients is needed to determine whether the proteinuria reduction is maintained, improves to remission or deteriorates to relapse.

In the two largest NS etiology patient groups, FSGS and $\mathrm{iMN}$, the majority of patients showed proteinuria reduction $\geq 50 \%$ following Acthar gel treatment, and the proteinuria reduction was significant. More than half of patients with FSGS showed partial remission and another quarter showed clinical response. Partial remission is a meaningful improvement for these patients, as significant improvement in kidney survival has been associated with partial remission of FSGS [14-16, 21]. Among patients with iMN, more than two-thirds of patients showed either complete or partial remission or clinical response to Acthar gel therapy. It has been stated that the recommended first-line therapy for patients with iMN, alkylating agents, should be restricted to patients who show a high risk of disease progression due to the toxicity associated with the agents [23]. Additionally, it has been shown that approximately $50 \%$ of patients with iMN with persistent high-grade proteinuria will progress to end-stage renal disease (ESRD) [1]. The current case series findings, outcomes from previous studies examining Acthar gel in patients with iMN, and the finding that risk of progression is significantly reduced with at least partial remission suggest that Acthar gel may provide an important treatment option for patients with treatmentresistant iMN [14, 15, 19, 22].

The remaining NS etiology patient groups each had $\leq 5$ patients, limiting conclusions about potential Acthar gel treatment efficacy. Proteinuria reduction to partial or complete remission was encouraging in patients with MCD or MLN and further study of Acthar gel therapy is warranted in these NS etiologies. Similarly, proteinuria response to Acthar gel therapy in 3 of the 5 patients with IgAN in our case series was consistent with prior single-case and small case series studies showing substantial proteinuria reduction $[14,15]$. Compared with the 4 patients with $\mathrm{DN}$ in our case series, a stronger proteinuria response to Acthar gel therapy has been demonstrated in patients with DN using a treatment regimen of $16 \mathrm{U}$ or $32 \mathrm{U}$ daily for 6 months [17].

Potential mechanisms of action of Acthar gel include steroid-independent effects through the melanocortin system and steroid-related effects [13, 24, 25]. Acthar gel in an animal model of progressive renal tubulointerstitial injury showed suppression of tubulointerstitial inflammation, tubular atrophy, and fibrosis through anti-inflammatory effects mediated by melanocortin receptor 1 (MC1R) on tubular epithelial cells [26]. MC1Rs have been shown in podocytes, glomerular endothelial cells, and mesangial cells, and an MC1R agonist resulted in significantly reduced proteinuria in the passive Heymann nephritis animal model [24]. Thus, Acthar gel steroid-independent effects may occur through melanocortin receptors, and more specifically MC1R, which may provide an explanation for the efficacy of Acthar gel in treatment-resistant and steroid-resistant patients $[24,26]$. Additionally, the cumulative dose of Acthar gel, through the dosing regimen and treatment duration, may be an important factor. Among iMN patients, those receiving a greater cumulative dose of Acthar gel $(2800 \mathrm{U})$ showed greater proteinuria reduction compared with lower cumulative doses (880 U and $1760 \mathrm{U})$ [19].

The current report is the largest case series to date to examine Acthar gel treatment of patients in real-world, clinical nephrology practices with an all-inclusive patient population. Strengths include the large patient sample with diverse NS etiologies and the inclusion of a majority of patients with prior NS treatment and with impaired renal function. The real-world clinical practice of Acthar gel treatment in patients with NS helps to elucidate the AEs that may be expected. Limitations include the small patient numbers in several of the NS etiologies, the retrospective design without a control group, and the possibility that concurrent therapy or long-term effects of prior immunosuppressive or cytotoxic therapy may have contributed to the proteinuria response during Acthar gel therapy in some patients. Patients in the current study were primarily White, which may limit the applicability of study findings in more racially diverse populations. Initiation of anti-proteinuria treatment was based on the treating clinician's judgment within the clinical management of their patient's changing NS symptoms. As a result, excluding patients with IgAN, 8 patients began treatment at a non-nephrotic proteinuria level $<3500 \mathrm{mg} /$ d. Proteinuria was used as a surrogate endpoint and the possible long-term benefit of Acthar gel in preventing ESRD was not examined. Additionally, longer treatment duration and follow-up may be needed for optimal treatment responses. The relapse rate following successful treatment with Acthar gel and possible use of other therapies post-Acthar gel treatment cessation are not yet known.

\section{Conclusion}

The current case series findings support a potential short-term benefit of Acthar gel therapy in patients with NS, particularly FSGS and iMN etiologies, and indicate Acthar gel treatment is well tolerated. Among the patients who completed $\geq 6$ months of treatment, $80 \%$ showed $\geq 30 \%$ proteinuria reduction, and almost twothirds showed $\geq 50 \%$ proteinuria reduction. The majority of patients who showed proteinuria reduction had failed 
prior immunosuppressive and cytotoxic therapies, and approximately half showed impaired renal function prior to Acthar gel treatment. These findings indicate that Acthar gel may meet an important treatment need in patients with NS that is treatment-resistant in response to first-line therapies or who are unable to tolerate firstline therapies and in patients with advanced disease. Future research is needed to determine whether patients who show a proteinuria clinical response without remission benefit from longer-term treatment and show continued clinical improvement. Further research using prospective, controlled trials with longer-duration treatment and follow-up assessments to examine different Acthar gel regimens and cumulative dose effects in varied-etiology NS is warranted.

\section{Availability of supporting data}

With the exception of total cholesterol, all raw data used in study summary analyses are provided in Tables 2, 3, 4, 5 and 6. Total cholesterol raw data are available on request.

\begin{abstract}
Abbreviations
ACEl: angiotensin-converting-enzyme inhibitor; ACR: American College of Rheumatology; $\mathrm{ACTH}$ : adrenocorticotropic hormone; $\mathrm{AE}$ : adverse event; ARB: angiotensin II receptor blocker; CNI: calcineurin inhibitor; DN: diabetic nephropathy; ESRD: end-stage renal disease; FGN: fibrillary glomerulonephritis; FSGS: idiopathic focal segmental glomerulosclerosis; IgAN: IgA nephropathy; iMN: idiopathic membranous nephropathy; KDIGO: kidney disease: improving global outcomes; MC1R: melanocortin receptor 1; MCD: minimal change disease; MLN: membranous lupus nephritis (SLE class V); MMF: mycophenolate mofetil; MPGN: membranoproliferative glomerulonephritis; NS: nephrotic syndrome; SCr: serum creatinine; SLE: systemic lupus erythematosus; UPCR: urine protein:creatinine ratio.

\section{Competing interests}

AM: Speaker for Mallinckrodt ARD Inc. (formerly Questcor). S M-D: Speaker for Mallinckrodt ARD Inc. (formerly Questcor). AS: Speaker for Mallinckrodt ARD Inc. (formerly Questcor). GT: Speaker for Mallinckrodt ARD Inc. (formerly Questcor). ASM: No competing interests.

AK: Advisor to Mallinckrodt ARD Inc. (formerly Questcor).
\end{abstract}

\section{Authors' contributions}

AM and AK contributed to study conception and design. All authors participated in data acquisition, data analysis, and data interpretation. Each author contributed important intellectual content during manuscript drafting or revision and accepts accountability for the overall work by ensuring that questions pertaining to the accuracy or integrity of any portion of the work are appropriately investigated and resolved. All authors read and approved the final manuscript.

\section{Acknowledgements}

The authors thank Dr. Sheldon M. Shore of Nephrology Consultants of GA for contributing patients to this study and Harvey Kushner, PhD, of the Biomedical Computer Research Institute for biostatistical analyses. The authors also thank Lynanne McGuire, PhD, of MedVal Scientific Information Services, LLC, for providing medical writing and editorial assistance. Funding to support the preparation of this manuscript was provided by Mallinckrodt ARD Inc. (formerly Questcor), to MedVal Scientific Information Services, LLC, Skillman, New Jersey. Mallinckrodt ARD did not have a role in the collection, analysis, or interpretation of the data; in writing the report; or in the decision to submit for publication. This manuscript was prepared according to the International Society for Medical Publication Professionals' "Good Publication
Practice for Communicating Company-Sponsored Medical Research: The GPP2 Guidelines."

\section{Author details}

${ }^{1}$ Nephrology Associates of Central Florida, 3885 Oakwater Circle, Orlando, FL 32806, USA. 'A Albany Medical College, Albany, NY, USA. ${ }^{3}$ HCA Inc. Physician Services, Salem, NH, USA. ${ }^{4}$ Lankenau Medical Center, Wynnewood, PA, USA. ${ }^{5}$ Nephrology Practice, 3366 NW Expressway, Bldg D, Suite 280, Oklahoma City, OK 73112, USA.

Received: 31 August 2015 Accepted: 15 March 2016

Published online: 31 March 2016

\section{References}

1. Kidney Disease: Improving Global Outcomes (KDIGO) Glomerulonephritis Work Group. KDIGO clinical practice guideline for glomerulonephritis. Kidney Int Suppl. 2012;2:139-274.

2. Beck L, Bomback AS, Choi MJ, Holzman LB, Langford C, Mariani LH, Somers MJ, Trachtman H, Waldman M. KDOQI US commentary on the 2012 KDIGO clinical practice guideline for glomerulonephritis. Am J Kidney Dis. 2013;62:403-41.

3. Segarra-Medrano A, Jatem-Escalante E, Agraz-Pamplona I, Carnicer-Caceres C, Ramos-Terrades N, Ostos-Roldan E, Quiles-Perez MT, Arbos-Via MA. Treatment of idiopathic focal segmental glomerulosclerosis: options in the event of resistance to corticosteroids and calcineurin inhibitors. Nefrologia. 2013:33:448-61.

4. Waldman M, Crew RJ, Valeri A, Busch J, Stokes B, Markowitz G, D'Agati V, Appel G. Adult minimal-change disease: clinical characteristics, treatment, and outcomes. Clin J Am Soc Nephrol. 2007;2:445-53.

5. Manno C, Torres DD, Rossini M, Pesce F, Schena FP. Randomized controlled clinical trial of corticosteroids plus ACE-inhibitors with long-term follow-up in proteinuric IgA nephropathy. Nephrol Dial Transplant. 2009;24:3694-701.

6. Reich HN, Troyanov S, Scholey JW, Cattran DC. Remission of proteinuria improves prognosis in IgA nephropathy. J Am Soc Nephrol. 2007;18:3177-83.

7. Hahn BH, McMahon MA, Wilkinson A, Wallace WD, Daikh DI, Fitzgerald JD, Karpouzas GA, Merrill JT, Wallace DJ, Yazdany J, Ramsey-Goldman R, Singh K, Khalighi M, Choi SI, Gogia M, Kafaja S, Kamgar M, Lau C, Martin WJ, Parikh S, Peng J, Rastogi A, Chen W, Grossman JM. American College of Rheumatology guidelines for screening, treatment, and management of lupus nephritis. Arthritis Care Res (Hoboken). 2012;64:797-808.

8. Radhakrishnan J, Moutzouris DA, Ginzler EM, Solomons N, Siempos II, Appel GB. Mycophenolate mofetil and intravenous cyclophosphamide are similar as induction therapy for class $V$ lupus nephritis. Kidney Int. 2010;77:152-60

9. Philibert D, Cattran D. Remission of proteinuria in primary glomerulonephritis: we know the goal but do we know the price? Nat Clin Pract Nephrol. 2008:4:550-9.

10. H.P. Acthar ${ }^{\oplus}$ Gel (repository corticotropin injection) [prescribing information] Hazelwood: Mallinckrodt ARD Inc.; 2015.

11. Rapoport M, McCrory WW, Barbero G, Barnett HL, Forman CW. Effect of corticotropin (ACTH) on children with the nephrotic syndrome. JAMA. 1951;147:1101-6.

12. Durand P, DeToni Jr E. Treatment of nephrotic syndrome in children. An Paediatr. 1955;185:225-35.

13. Gong R. Leveraging melanocortin pathways to treat glomerular diseases. Adv Chronic Kidney Dis. 2014;21:134-51.

14. Bomback AS, Tumlin JA, Baranski J, Bourdeau JE, Besarab A, Appel AS, Radhakrishnan J, Appel GB. Treatment of nephrotic syndrome with adrenocorticotropic hormone (ACTH) gel. Drug Des Devel Ther. 2011;5:147-53.

15. Bomback AS, Canetta PA, Beck Jr LH, Ayalon R, Radhakrishnan J, Appel GB. Treatment of resistant glomerular diseases with adrenocorticotropic hormone gel: a prospective trial. Am J Nephrol. 2012;36:58-67.

16. Hogan J, Bomback AS, Mehta K, Canetta PA, Rao MK, Appel GB, Radhakrishnan J, Lafayette RA. Treatment of idiopathic FSGS with adrenocorticotropic hormone gel. Clin J Am Soc Nephrol. 2013;8:2072-81.

17. Tumlin JA, Galphin CM, Rovin BH. Advanced diabetic nephropathy with nephrotic range proteinuria: a pilot study of the long-term efficacy of subcutaneous ACTH gel on proteinuria, progression of CKD, and urinary levels of VEGF and MCP-1. J Diabetes Res. 2013;2013:489869. 
18. Watson MJ. Membranous glomerulopathy and treatment with Acthar ${ }^{6}$. a case study. Int J Nephrol Renovasc Dis. 2013;6:229-32.

19. Hladunewich MA, Cattran D, Beck LH, Odutayo A, Sethi S, Ayalon R, Leung $\mathrm{N}$, Reich $\mathrm{H}$, Fervenza FC. A pilot study to determine the dose and effectiveness of adrenocorticotrophic hormone (H.P. Acthar ${ }^{\otimes}$ Gel) in nephrotic syndrome due to idiopathic membranous nephropathy. Nephrol Dial Transplant. 2014;29:1570-7.

20. Lieberman KV, Ettinger L, Picarelli C: Adrenocorticotropic hormone for steroid-resistant and oral steroid-intolerant children with minimal change nephrotic syndrome. J Clin Pediatr Nephrol 2014, 2: http://www. jpnephrology.com/index.php/FIRST/article/view/64.

21. Troyanov S, Wall CA, Miller JA, Scholey JW, Cattran DC. Focal and segmental glomerulosclerosis: definition and relevance of a partial remission. J Am Soc Nephrol. 2005;16:1061-8.

22. Troyanov S, Wall CA, Miller JA, Scholey JW, Cattran DC. Idiopathic membranous nephropathy: definition and relevance of a partial remission. Kidney Int. 2004;66:1199-205.

23. Hofstra JM, Fervenza FC, Wetzels JF. Treatment of idiopathic membranous nephropathy. Nat Rev Nephrol. 2013;9:443-58.

24. Lindskog A, Ebefors K, Johansson ME, Stefansson B, Granqvist A, Arnadottir M, Berg AL, Nystrom J, Haraldsson B. Melanocortin 1 receptor agonists reduce proteinuria. J Am Soc Nephrol. 2010;21:1290-8.

25. Gong R. The renaissance of corticotropin therapy in proteinuric nephropathies. Nat Rev Nephrol. 2012;8:122-8.

26. Gong R, Dworkin LD. Adrenocorticotropin (ACTH) gel suppresses renal tubulointerstitial inflammation and injury by direct stimulation of the melanocortin 1 receptor (MC1R) [abstract]. J Am Soc Nephrol. 2011;22(suppl):136A.

\section{Submit your next manuscript to BioMed Central and we will help you at every step:}

- We accept pre-submission inquiries

- Our selector tool helps you to find the most relevant journal

- We provide round the clock customer support

- Convenient online submission

- Thorough peer review

- Inclusion in PubMed and all major indexing services

- Maximum visibility for your research

Submit your manuscript at www.biomedcentral.com/submit 